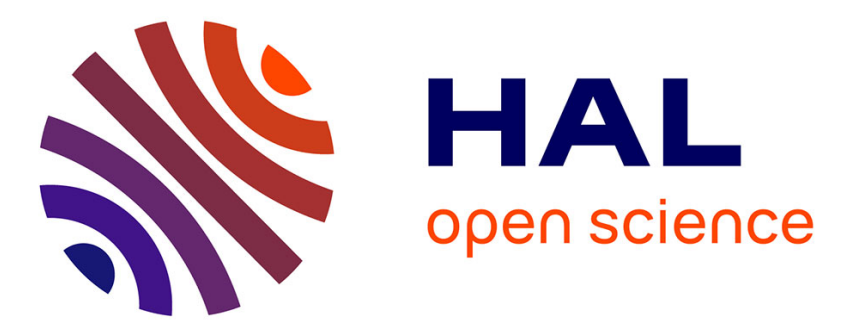

\title{
Comparison of corneal endothelial mosaic according to the age: the corimmo $3 \mathrm{D}$ project
}

Klervi Rannou, Emmanuel Crouzet, Caroline Ronin, Patricio Guerrero, Gilles Thuret, Philippe Gain, Jean-Charles Pinoli, Yann Gavet

\section{To cite this version:}

Klervi Rannou, Emmanuel Crouzet, Caroline Ronin, Patricio Guerrero, Gilles Thuret, et al.. Comparison of corneal endothelial mosaic according to the age: the corimmo 3D project. Innovation and Research in BioMedical engineering, 2016, 37 (2), pp.124-130. 10.1016/j.irbm.2016.03.004 . hal01365604

\section{HAL Id: hal-01365604 https://hal.science/hal-01365604}

Submitted on 15 Sep 2016

HAL is a multi-disciplinary open access archive for the deposit and dissemination of scientific research documents, whether they are published or not. The documents may come from teaching and research institutions in France or abroad, or from public or private research centers.
L'archive ouverte pluridisciplinaire HAL, est destinée au dépôt et à la diffusion de documents scientifiques de niveau recherche, publiés ou non, émanant des établissements d'enseignement et de recherche français ou étrangers, des laboratoires publics ou privés. 


\title{
Comparison of corneal endothelial mosaic according to the age : the CorImMo 3D project
}

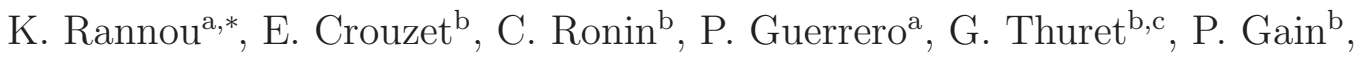 \\ J.C. Pinoli ${ }^{\mathrm{a}}$, Y. Gavet ${ }^{\mathrm{a}}$ \\ ${ }^{a}$ École Nationale Supérieure des Mines de Saint-Étienne, LGF UMR CNRS 530\%, \\ 158 cours Fauriel, CS 62362, 42023 Saint-Étienne, France \\ ${ }^{b}$ Corneal Graft Biology-Engineering and Imaging Laboratory, EA2521-Federative \\ Institute of Research in Sciences and Health Engineering-Faculty of Medicine-Jean \\ Monnet University, Saint-Etienne, France \\ ${ }^{c}$ Institut Universitaire de France, Boulevard St Michel, Paris, France
}

\begin{abstract}
Aim: The human corneal endothelium is a monolayer of flat hexagonal cells. It is a nearly regular hexagonal tessellation during the first years of life, but with age, becomes less regular in shape and size. The aim is to evaluate geometrically the age of an endothelial mosaic.

Material and methods: Segmented endothelial mosaics of healthy subjects of different age groups are compared by morphological criteria. The mosaics are studied according to their age group (decades), their age and their location (center or mid-periphery of the cornea). The measures used are : the cell density, the Ripley's $L$ function and the cell area and perimeter density.

Results: These measures point out the endothelial cell density decrease, the cell area, perimeter and diameter increase, the cell heterogeneity increase, and the differences between central and mid-peripheral cells increases with
\end{abstract}

\footnotetext{
*Corresponding author

Email address: klervi.rannou@emse.fr (K. Rannou)
} 
age.

Conclusion: These measures are able to characterize healthy mosaics.

Keywords: corneal endothelium, cell morphology, Ripley's function, area density, perimeter density

\section{Introduction}

2 The human corneal endothelium is a monolayer of flat hexagonal cells, 3 which do not regenerate and are responsible for the maintenance of the cornea 4 transparency. When the number of endothelial cells (ECs) is too low, the 5 cornea becomes edematous, causing irreversible loss of vision that can only 6 be treated by a corneal graft. The donor cornea brings numerous new func7 tioning ECs into the recipient eye. Because of their location at the most 8 posterior layer of this transparent tissue, ECs can be visualized in vivo using 9 a specular microscope using the light reflected by the interface between ECs and the liquid that fills the anterior chamber of the eye. Similarly, they can be observed ex vivo during corneal storage using a transmitted light microscope 
year during adulthood [1]. Nevertheless, in healthy corneas, the number of ECs remains always high enough to maintain corneal clarity even in centenarians. This important notion of endothelial reserve disappears when diseases or traumatisms alter the endothelium. In these situations, decrease of ECD and changes in pleomorphism (i.e. shape variability) and polymorphism (i.e. size variability) can be dramatically accelerated, ultimately leading to corneal opacification requiring corneal graft.

In eye banks, donor corneas are stored and strictly controlled in order to verify if they are suitable for corneal graft. Quality of the endothelium is the main criterion to decide whether a cornea can be grafted or must be destroyed. At present, ECD is the only quantitative parameter used. A threshold under which a cornea is unsuitable for graft determines the fate of each donor cornea. It is usually of 2000 cells $/ \mathrm{mm}^{2}$ for corneas destined to penetrating keratoplasty (replacement of the whole thickness of the central cornea, constituting the gold standard and the most frequent technique worldwide) and 2400 cells $/ \mathrm{mm}^{2}$ for corneas destined to posterior endothelial graft (selective replacement of the endothelium, requiring preparation of a thin posterior lamellae that can be slightly harmful to the ECs, explaining the higher threshold). For CV and hexagonality that can be measured with image analysis [2], their influence on the post graft endothelial survival has never been studied. They are at present used as additional criteria to help qualifying corneas with ECD near the threshold.

In order to better explain endothelial aging and some of the most frequent clinical situations (ECD decrease in Fuchs corneal endothelial dystrophy, the most frequent primary endothelial dystrophy, and after corneal grafts), new 
methods to qualify the endothelial mosaic, using geometrical and morphological criteria, are studied. The aim is to establish an original mathematical model of the human corneal endothelium. In the present work, three measures of the cell size variability are presented: the Ripley's L function and the area and perimeter cells densities. These mathematical parameters are used to assess the age of an endothelial mosaic of healthy corneas.

\section{Material and methods}

\subsection{Source of endothelial images}

Images were taken using a small field non-contact specular microscope (SP 3000, Topcon, Tokyo, Japan) (Fig.1). In 10 age groups (from 0 to 10 years old, 11 to 20,21 to $30, \ldots$, and 91 to 100), images of healthy eyes of 5 subjects that were taken during routine examination, were selected. Images were anonymised and patients could not be recognized from the pictures.

ECD is not homogeneous on the whole endothelium, it progressively decreases toward center $([4,3])$. For each eye, five images were therefore taken in the central, temporal, nasal, superior and the inferior zones of the endothelium, by asking the patient to focus on each of the 5 LEDs placed on the microscope to orientate the eyeball. The 4 non central positions were localized 3 to 4 millimeter from the center, that is to say not in the extreme periphery of the cornea. As non-contact specular microcope have a narrow field of view, the acquisition of 5 images distributed on the corneal surface is the usual protocol used in routine to increase the sampling and obtain a more representative analysis. Each image was manually segmented by an expert using ImageJ (Fig.2). 


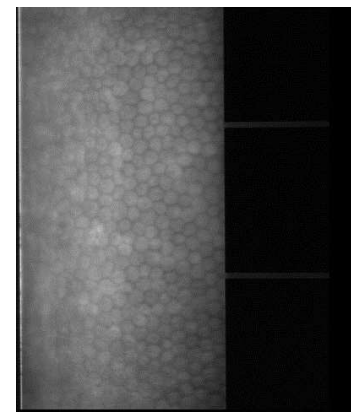

(a) Central

4-year-old patient

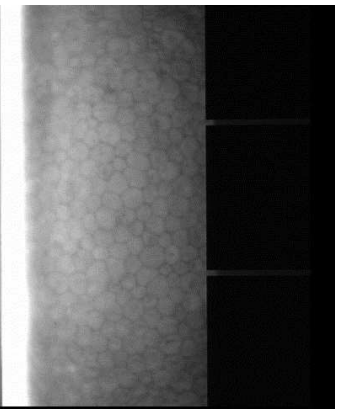

(b) Central

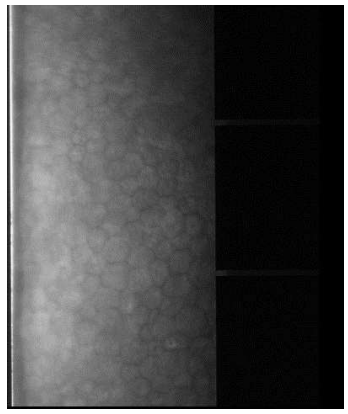

(c) Central

41-year-old patient 92-year-old patient

Figure 1: Representative images of the endothelial mosaic taken using a small field noncontact specular microscope.

\section{0}

71

\subsection{Ripley's L function}

The Ripley's $L$ function (RLF) is used to analyze the spatial distribution of a collection of points. The RLF counts the mean number of mass centers at a given distance from another mass center $[5,6]$.

Let $P=\left\{p_{1}, p_{2}, \ldots, p_{N}\right\}$ be a collection of $N$ points in the image $I$, considered as a bounded region of $\mathbb{R}^{2}$, and let $A$ be the area of $I$.

An estimator of the RLF is given, for all $r \geq 0$, by:

$$
\hat{L}(r)=\sqrt{\frac{A}{\pi N^{2}} \sum_{i=1}^{N} \sum_{j \neq i} \delta_{i j}(r)},
$$

where $\delta_{i j}(r)$ is equal to 1 if the distance between the points $p_{i}$ and $p_{j}$ is less than $r$, and 0 otherwise.

The RLF is compared to the stationary Poisson point process one, that serves as a measure of complete randomness and lack of interaction. In the case of a Poisson point process, $L(r)=r$ for all distance $r$. Moreover, for 


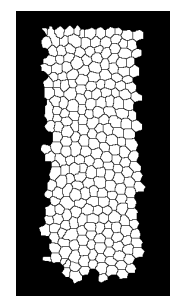

(a) Central

4-y.o. patient

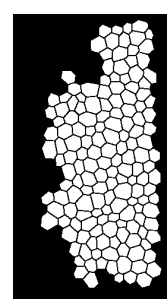

(f) Central

41-y.o. patient

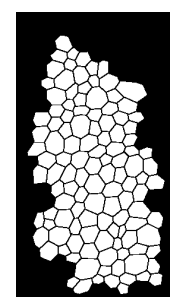

(k) Central

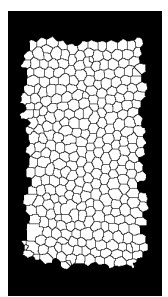

(b) Inferior

4-y.o. patient

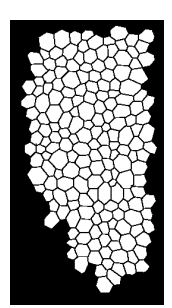

(g) Inferior

41-y.o. patient

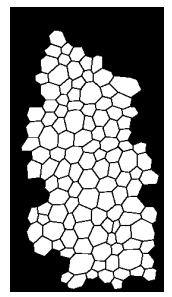

(l) Inferior

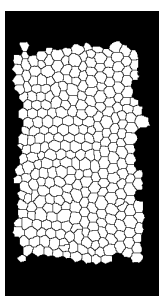

(c) Nasal

4-y.o. patient

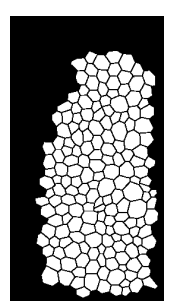

(h) Nasal

41-y.o. patient

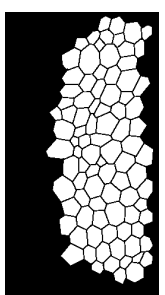

(m) Nasal

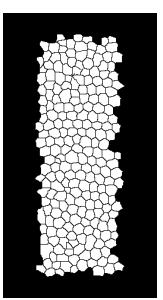

(d) Superior

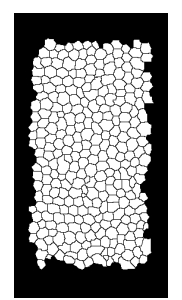

(e) Temporal 4-y.o. patient

92-y.o. patient 92-y.o. patient 92-y.o. patient 92-y.o. patient 92-y.o. patient

Figure 2: Representative segmented endothelial mosaics of the central, inferior, nasal, superior and temporal zones of the right eye of three patients. They illustrate that cell area, the polymorphism and pleomorphism increase with age. 


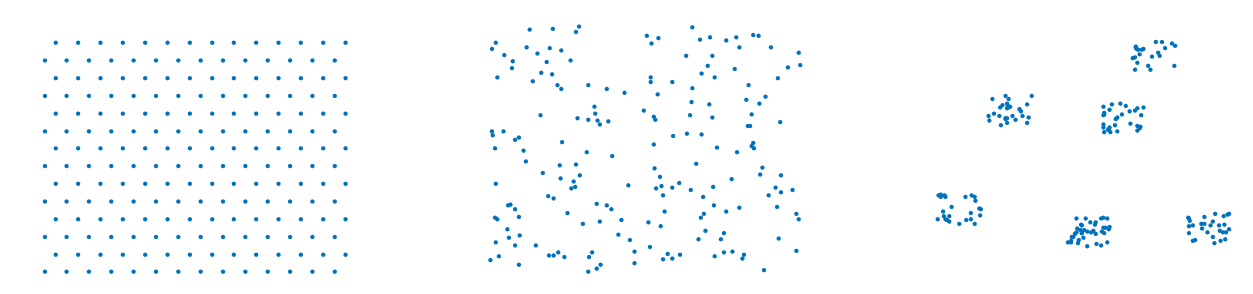
(a) Regular
(b) Poisson point process
(c) Clustered

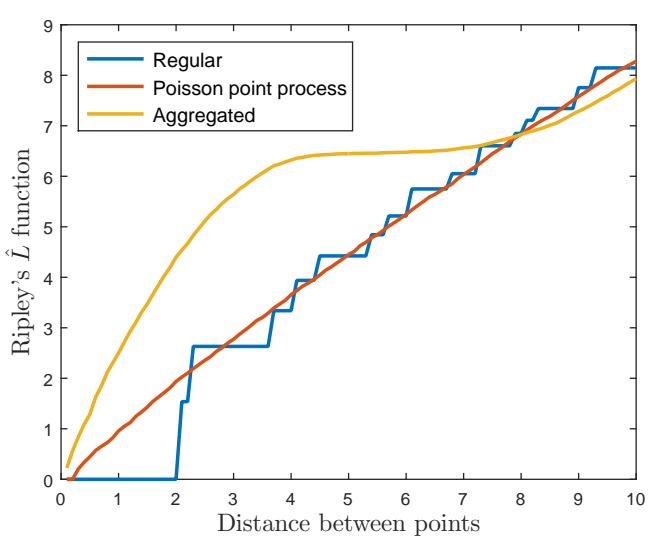

(d) Ripley's $\hat{L}$ function

Figure 3: Three collections of points and their Ripley's $\hat{L}$ function. (a) is a regular point collection, $\hat{L}$ is a step function and for small distances, $\hat{L}(r)<r$. (b) is a realization of a Poisson point process, $\hat{L}$ is linear. (c) are clustered points, $\hat{L}(r)>r$.

82 small distances, $L(r)<r$ indicates regularity and $L(r)>r$ aggregation 83 (Fig.3).

In the case of the endothelial mosaic, the points considered are the mass ${ }_{85}$ centers of the ECs. The RLF provides information about the spatial distribution of the cells mass centers, and consequently about the distance between 87 cells mass centers, that is to say their diameters. 


\subsection{Area and perimeter density}

Another way to study the cell size variability according to the age, is to use the area and perimeter density of ECs.

Let $\left(a_{1}, \ldots, a_{k}\right)$ be a sample of observations : cell area or perimeter (of a patient, or an age group, etc.). The density function $f$ of this sample is estimated by the kernel density estimator [7, 8], which is:

$$
\hat{f}(x):=\frac{1}{b k} \sum_{i=1}^{k} K\left(\frac{x-a_{i}}{b}\right),
$$

where $K($.$) is a kernel function and b>0$ is the smoothness parameter, called bandwidth, proportional to $k^{-\frac{1}{5}}$. The kernel function used is the Epanechnikov kernel function [9].

A kernel density estimator is used rather than an histogram, because the histogram method have fixed classes whereas the kernel estimator is mobile and centered on each observation.

\section{Results}

\subsection{Endothelial cell density}

First, the mean ECD per age group and per patient is calculated over all images of an age group or patient (Fig.4a and 4b). As expected, ECD decreased with age and the variability between patients of the same age class increased (the coefficient of variation computed over all images of an age group increases, Fig.4c).

\subsection{Ripley's L function}

The $\hat{L}$ function was calculated for the cell mass centers of each segmented image of an age group. The mean $\hat{L}$ function over all images of an age group 


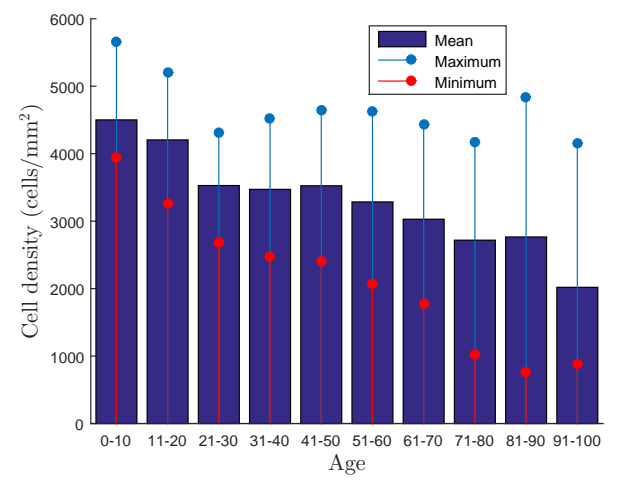

(a) ECD per age group

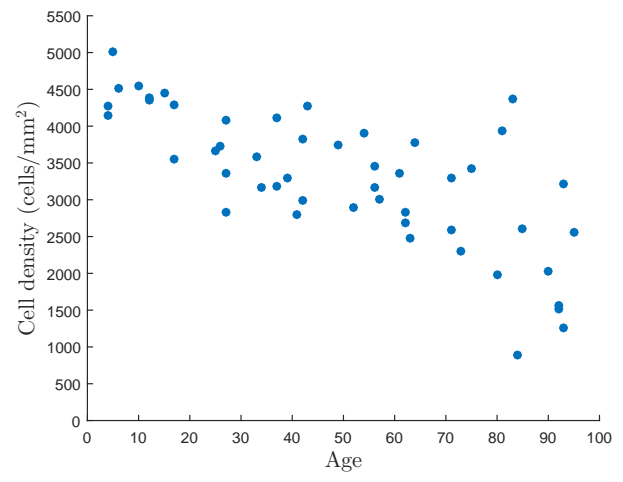

(b) ECD per patient

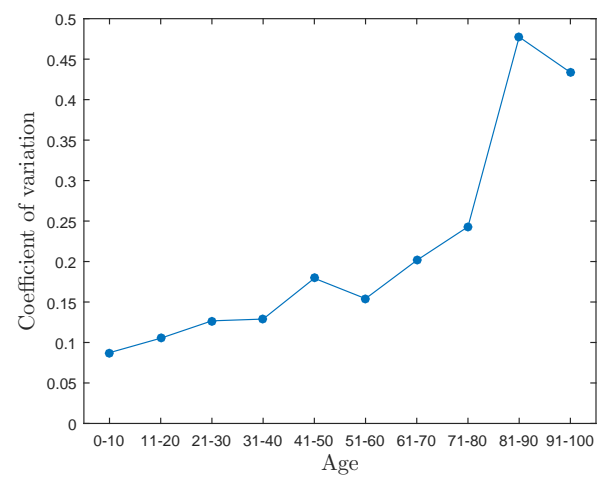

(c) Coefficient of variation per age group

Figure 4: (a) Mean, minimum and maximal endothelial cell density of all images of each age group, (b) mean cell density for each patient, and (c) the coefficient of variation for each age group. 
was then represented graphically, and compared to the one of realizations of Poisson point processes (Fig.5a). For all age groups, the mean $\hat{L}$ function is null for small distances and become non null earlier for the youngest group, meaning that the smallest distance between mass centers increases with age. Oscillations of the mean RLF were marked for the youngest age groups and decreased with age, indicating that homogeneity in cell diameters decreased with age. Furthermore, the first rebound for the youngest age groups indicates the maximum distance between mass centers of neighbor cells.

For 3 age groups (young: 0-10 years old, middle age: 41-50 years old, and elderly age: 91-100 years old), we compared the RLF of the ECs from the center of the cornea with the mean RLF of the 4 images taken in the mid periphery of the cornea (Fig.5b).

To quantify the difference between two curves, the error in percent was compute between the curve of the central $\mathcal{C}_{1}$ and the mid peripheral cells $\mathcal{C}_{2}$ :

$$
E\left(\mathcal{C}_{1}, \mathcal{C}_{2}\right)=\frac{\left\|C_{1}-C_{2}\right\|_{1}}{\frac{1}{2}\left\|C_{1}+C_{2}\right\|_{1}} \times 100
$$

where $\|.\|_{1}$ is the $l_{1}$ norm (also called Manhattan or Taxicab norm). No big difference was observed between center and mid periphery $(E<1 \%)$, except for the elderly age group (Fig.6), but it is probably due to the small number of cells per image for some elderly patients.

\subsection{Area and perimeter density}

The standard deviation of the cell area and perimeter mean estimate density progressively increases with age (wider dispersion around the peak), and indicates a gradual increase in heterogeneity (Fig.7). The function $E$ (3) 


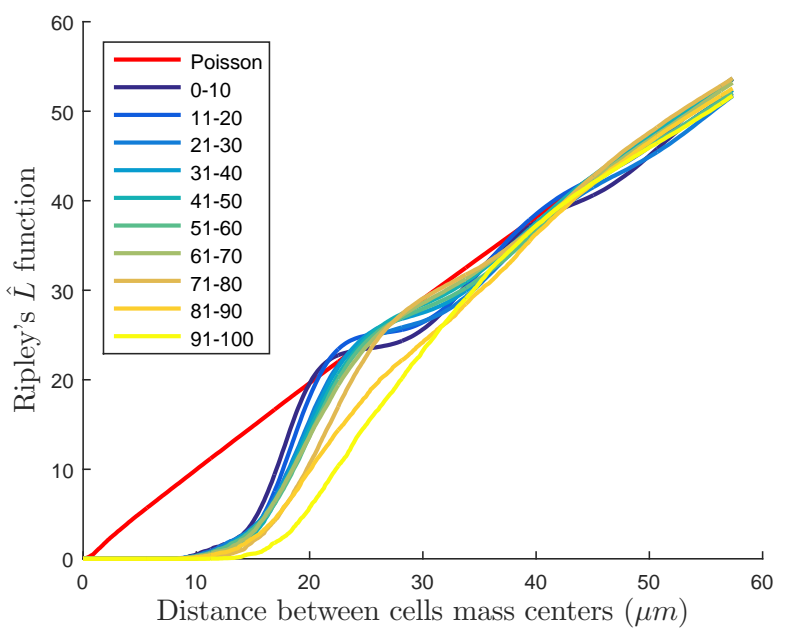

(a)

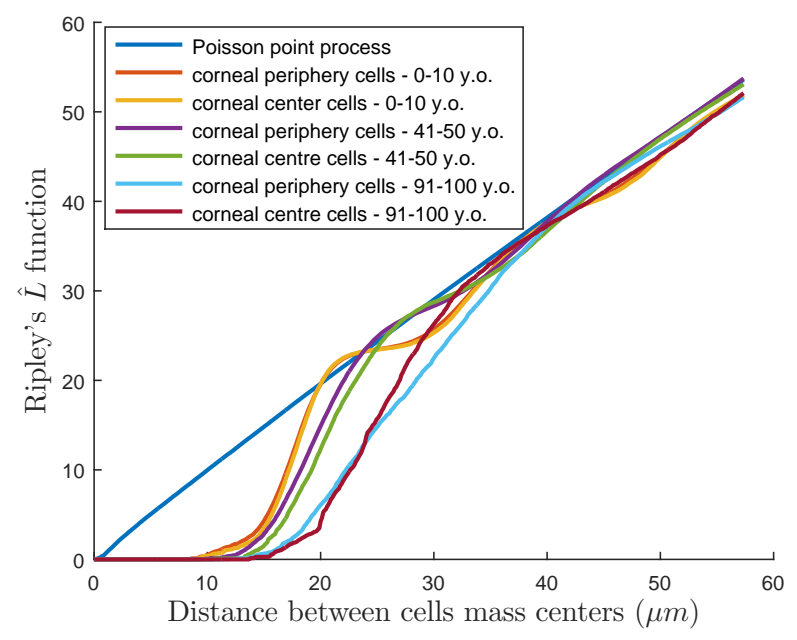

(b)

Figure 5: The mean Ripley's $\hat{L}$ function for realizations of a Poisson point process and for endothelial mosaics. The mean $\hat{L}$ function (a) for each age group and (b) for cells observed in the center versus in the mid periphery of the cornea in 3 age groups. 


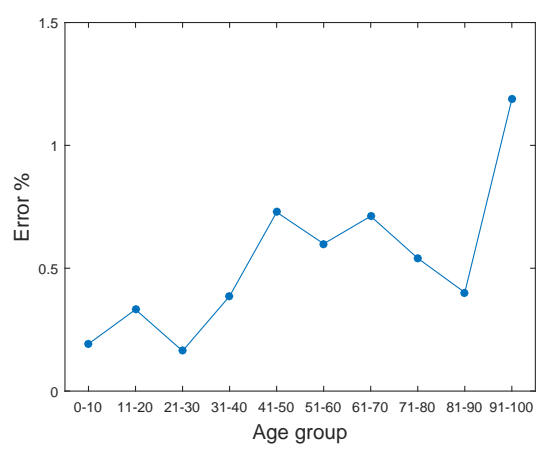

Figure 6: Error between the mean Ripley's $\hat{L}$ function of center and mid peripheral endothelial cells.

was calculated for each patient between his density mean estimate and his age group density mean estimate, to quantify the inter-individual variability in each age group, and showed the increase of inter-individual variability (Fig.8a).

Next, the cell area and perimeter estimate density of the central cells was compared to the mean estimates densities of the mid peripheral cells for 3 age groups (Fig.7e-7f). For the two oldest age groups, the mean cell area and perimeter (density peak) is higher in the central cells than in the mid periphery of the cornea, indicating that, with age, the central cells become bigger than in the mid periphery. The computation of the $E$ function, between densities mean estimates of central and mid peripheral ECs, pointed out these increases of differences between center and mean periphery with age (Fig.8b). 


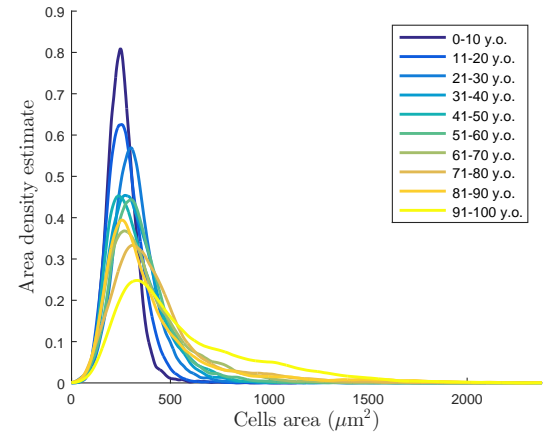

(a) Area

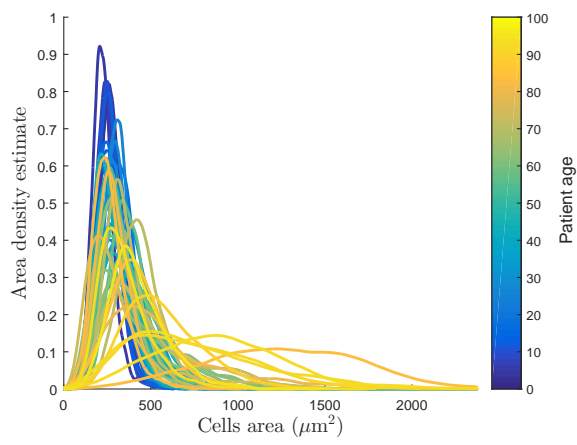

(c) Area

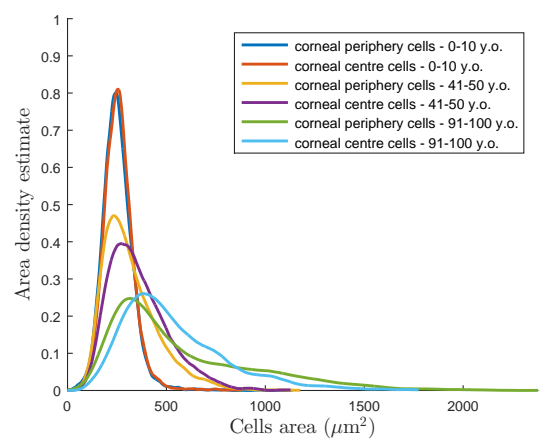

(e) Area

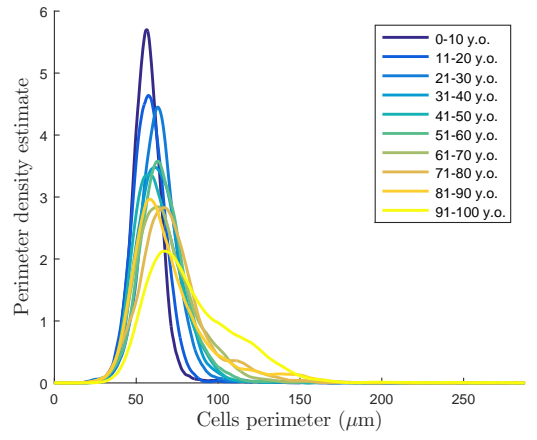

(b) Perimeter

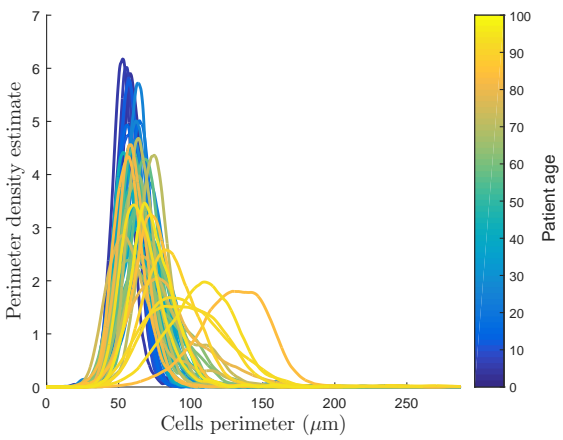

(d) Perimeter

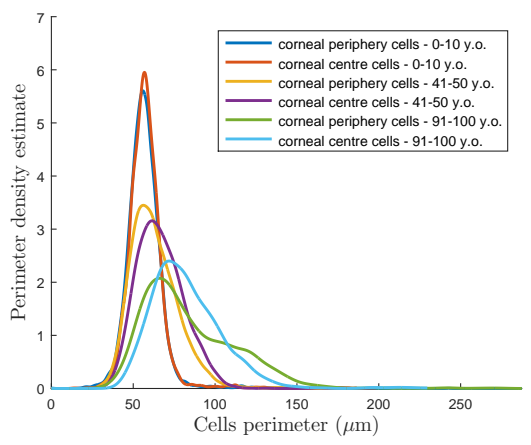

(f) Perimeter

Figure 7: Cells area and perimeter density mean estimate. (a)-(b) for each age group, (c)(d) for each patient, and (e)-(f) for cells observed in the center versus in the mid periphery of the cornea in 3 age groups. 


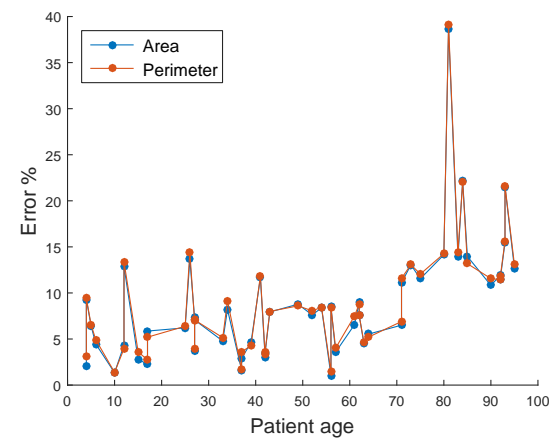

(a)

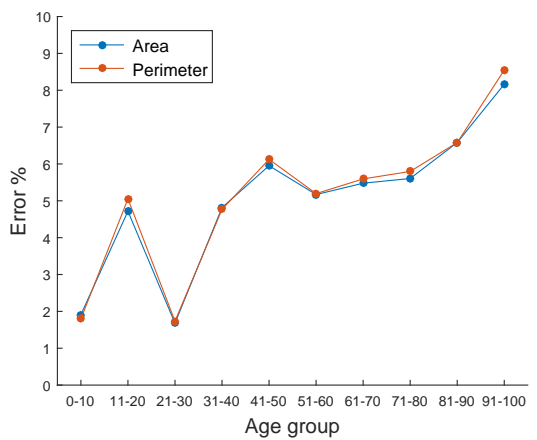

(b)

Figure 8: Error between area and perimeter densities mean estimates : (a) between the mean age group curve and each patient curve, to quantify the variability between patients, and (b) for each age group, between the curves of the center and the mid peripheral endothelial cells.

\section{Discussion}

The number of subjects is quiet low per decade, and for the oldest groups, the small field of observation of the non-contact microscope was an obstacle because it greatly limited the number of entirely visible big ECs. Therefore, a great number of ECs were available to analyze the endothelial mosaic per decade, but not to study them image per image or to compare central cells to outlying cells for some subjects. Repeating the analysis with more subjects and using wide field digital contact specular microscopy images [10] would validate and improve the accuracy of our measurements. Further works are ongoing to constitute a bank of images of wide field digital contact specular microscopy images.

Despite the time-consuming task, the segmentation have been made manually by an expert to avoid the bias induced by automatic segmentation 
methods, and in order that the segmented endothelial mosaics serve as reference.

In this preliminary study, it has been shown that the ECD, the RLF and the area and perimeter density estimate are able to characterize the human corneal endothelial mosaic changes occurring with age. These measures point out the differences according to the age : they find the same well-known increase in cell area (diameter and perimeter) and increase in cell heterogeneity, they point out that inter-individual variability increases and that a difference between size of ECs from the central (bigger) and the mid-peripheral cornea appears with age. The time needed to compute all these measures is quite low : the mean time for one view is 0.63 seconds (the maximum time is 1.32 seconds).

\section{Conclusion}

Original geometrical and morphological criteria are able to characterize the healthy human corneal endothelial mosaic. Works are now ongoing to study other parameters like the number of neighbor cells, morphometric criteria by using shape diagrams [11], etc. Applied to the most frequent pathological endothelial modifications (ECs loss after corneal grafts and in Fuchs corneal endothelial dystrophy), these new criteria could bring new insights in their physiopathology.

\section{Acknowledgments}

The authors wish to thank the French National Research Agency for financial support (ANR-12-TECS-004, CorImMo 3D). 


\section{References}

[1] W. M. Bourne, L. R. Nelson, D. O. Hodge, Central corneal endothelial cell changes over a ten-year period., Invest Ophth Vis Sci 38 (3) (1997) $779-82$

[2] S. Acquart, P. Gain, M. Zhao, Y. Gavet, A. Defreyn, S. Piselli, et al., Endothelial morphometry by image analysis of corneas organ cultured at 31 degrees c., Invest Ophth Vis Sci 51 (3) (2010) 1356-64.

[3] B., Schimmelpfennig, Direct and indirect determination of nonuniform cell density distribution in human corneal endothelium, Invest Ophth Vis Sci 25 (2) (1984) 223-9.

[4] Z. He, N. Campolmi, P. Gain, B. M. Ha Thi, J.-M. Dumollard, S. Duband, et al., Revisited microanatomy of the corneal endothelial periphery: new evidence for continuous centripetal migration of endothelial cells in humans, Stem Cells 30 (11) (2012) 2523-34.

[5] B. D. Ripley, Modelling spatial patterns, J. R. Stat. Soc. Ser. B Stat. Methodol. (1977) 172-212.

[6] M. N. M. vanLieshout, A. J. Baddeley, A nonparametric measure of spatial interaction in point patterns, Stat. Neerl. 50 (3) (1996) 344-61.

[7] M. Rosenblatt, Remarks on some nonparametric estimates of a density function, Ann. Math. Stat. 27 (3) (1956) 832-7.

[8] E. Parzen, On estimation of a probability density function and mode, Ann. Math. Stat. (1962) 1065-76. 
[9] V. A. Epanechnikov, Non-parametric estimation of a multivariate probability density, Theor Probab Appl+ 14 (1) (1969) 153-8.

[10] G. Hor, Y. Gavet, A. Bernard, C. Urrea, P. Gain, G. Thuret, Digitalization of a wide field contact specular microscope leads to better performances, 2016. Manuscript submitted for publication.

[11] K. Rannou, Y. Gavet, J.-C. Pinoli, Characterization of the corneal endothelial mosaic and comparison with simulated tessellations modeled with gaussian random fields, in: The International Conference on Quality Control by Artificial Vision 2015, International Society for Optics and Photonics, 2015. 